\title{
Features of Phage Therapy in Terms of Bioengineering
}

\author{
Krylov VN*, Bourkaltseva MV, Pleteneva EA and Krylov SV \\ Mechnikov Research Institute for Vaccines and Sera, Russia \\ *Corresponding author: Krylov VN, Mechnikov Research Institute for Vaccines and Sera, Moscow, Russia
}

\begin{tabular}{|c|c|}
\hline ARTICLE INFO & ABSTRACT \\
\hline $\begin{array}{l}\text { Received: November 25, } 2019 \\
\text { Published: December 03, } 2019\end{array}$ & Keywords: Phage Therapy; Phages Transposons; Phage Mixtures \\
\hline $\begin{array}{l}\text { Citation: Krylov VN, Bourkaltseva MV, } \\
\text { Pleteneva EA, Krylov SV. Features of Phage } \\
\text { Therapy in Terms of Bioengineering. Bi- } \\
\text { omed J Sci \& Tech Res 23(3)-2019. BJSTR. } \\
\text { MS.ID.003914. }\end{array}$ & \\
\hline
\end{tabular}

\section{Opinion}

The bioengineering concept is based on the traditional, common for any engineering approach, assessment of sensitivity to cost and practicality of the solutions found. In this regard, while traditional engineering uses physics and mathematics for the analysis, design and manufacture of non-living instruments, structures and processes, biological engineering uses mainly the rapidly developing field of molecular biology to study and proceed in manipulations with living organisms. Based on the generally accepted definition of bioengineering as finding the feasibility and practical use of scientific knowledge to solve current problems with cost-effective use, phage therapy can be considered as an excellent example of the application of this concept. It must be admitted that the recent critical rethinking of the possibility of the practical use of phage therapy in Western medicine (the concept of "Magistral Phage") [1] although it has a very limited character being associated with a prerequisite for patient consent, is nevertheless an outstanding success for Western countries.

In Russia, phage therapy being introduced by Felix D Herrell, one of the pioneers of discovery and study of bacterial viruses is in active application from the early 30 s of the last centuries. To what extent is phage therapy consistent with the concept of bioengineering? The main essence of phage therapy can be considered as a bioengineering process accomplished with the final purpose to provide an affordable and effective influence on the infectious process caused by a bacterial pathogen with use of bacterial viruses - bacteriophages in the absence of acceptable chemical antibiotics. In phage therapy as a composite tool of bioengineering, not only does the object- infecting s particle of a bacteriophage of a certain species change (with transition to a replicative form), but there is the possibility of additional complicated interspecific and intraspecies interactions not only between therapeutic bacteriophages, but also with prophages - temperate bacteriophages, which often present in pathogenic bacteria in inactive form. These interactions can affect not only the end result of antibacterial therapy, but also, in general, the patient's health status. It should be noted that in this case we are talking about truly virulent bacteriophages, activity of which causes the obligatory death of pathogenic bacterial cell as a result of the development of infecting phages. Thus, phage therapy has a number of fundamental differences from antibiotic therapy from the point of view of the bioengineering approach. First of all, for its full effectiveness, it is required to ensure the effectiveness of all stages of phage development during the propagation of the active principle - the phage genome - during infection with the resulting expression of the necessary functions (killing and lysis of bacteria with the release of viable offspring). It is assumed that, in the ideal case, the development of a bacteriophage in bacteria sensitive to it will lead to the exhaustion of a pool of pathogenic bacteria (in a wound or in an infected organ). In the case of chronic infections, including lungs, serious problems may arise with the 
need to quickly update therapeutic phage mixtures. However, there are a number of negative factors, compared with antibiotic therapy.

a) Very high phage specificity (in some cases, only a certain strain of pathogenic bacteria can be lysed).

b) The inability to stop the process of reproduction of phages and lysis of bacteria at will.

c) With a massive lysis of bacteria in the focus of infection, especially closed (like the lungs), the release of products that can exhibit a general toxic effect is inevitable.

d) It is possible that even related phage interactions occur during therapy, which can cause unwanted effects.

As an example, we can consider the possible (and yet unstudied) interaction of two related phages - D3112 (the moderate phage transposon Pseudomonas aeruginosa [2] and the virulent phage PA10 [3]. At the left end of the PA10 phage genome, there is no 5 $\mathrm{kb}$ fragment of the genome available D3112: It is possible that this difference is due to the loss of the ability of PA10 to transpose and the emergence of the ability to infect and multiply in bacteria of species unrelated to P. aeruginosa and other species [4]. How this can affect the possibility of genetic exchanges of phages and their interaction during therapy on the evolution of infectious bacteria during the course of the disease remains unclear. In addition, the almost inevitable occurrence of the rotation of infectious strains during nosocomial infections for chronic patients significantly limits the therapeutic effect. Much will depend on the ability of the technology to quickly create a mixture for the permanent treatment of chronic infection and its rapid modification during therapy. It is difficult to foresee in what ways the implementation of "magistral phage therapy" will develop.

It is possible that the increasing cost of repeated acts of phototherapy can become a limiting factor in therapy according

\section{ISSN: 2574-1241}

DOI: $10.26717 /$ BJSTR.2019.23.003914

Krylov VN. Biomed J Sci \& Tech Res

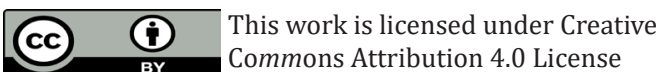

Submission Link: https://biomedres.us/submit-manuscript.php to the magistral phage principle. Of course, the construction of a therapeutic mixture will be seriously complicated with multispecies bacterial infection. In this sense, the design and industrial production of regionally adapted phage mixtures that are active against the most common types of hospital pathogens, adopted in Russia, seems more preferable. Nevertheless, the transition to the recognition of phage therapy in the West as a promising antibacterial therapy and the ideas associated with the development of approaches for the intravenous medication of phages for treatment are well founded [5]. We do not discuss here the more distant prospects of creating special phage variants not recognized by defense systems, and other bioengineering approaches, although here we can also expect the development of new promising areas of phage therapy [6].

\section{References}

1. Pirnay JP, Verbeken G, Ceyssens PJ, Huys I, De Vos D (2018) The Magistral Phage. Viruses 10(2): E64.

2. Krylov VN, Bogush VG, Shapiro J (1980) Pseudomonas aeruginosa bacteriophages whose DNA is similar in structure to that of phage Mu1. 1.General description, localization of sites sensitive to endonucleases in DNA and structures of homoduplexes of phage D3112. Genetika 16(5): 824-832.

3. Kim S, Rahman M, Kim J (2012) Complete genome sequence of Pseudomonas aeruginosa lytic bacteriophage PA10 which resembles temperate bacteriophage D3112. J Virol 86(6): 3400-3401.

4. Kim S, Rahman M, Seol SY, Yoon SS, Kim J (2012) Pseudomonas aeruginosa bacteriophage PA1Ø equires type IV pili for infection and shows broad bactericidal and biofilm removal activities. Appl Environ Microbiol. 78(17): 6380-6385.

5. Vitiello CL, Merril CR, Adhya S (2005) An amino acid substitution in a capsid protein enhances phage survival in mouse circulatory system more than a 1000-fold. Virus Res 114(1-2):101-103.

6. Yibao Chen, Himanshu Batra, Junhua Dong, Cen Chen, Venigalla B Rao (2019) Genetic Engineering of Bacteriophages Against Infectious Diseases. Frontiers in Microbiology 10.

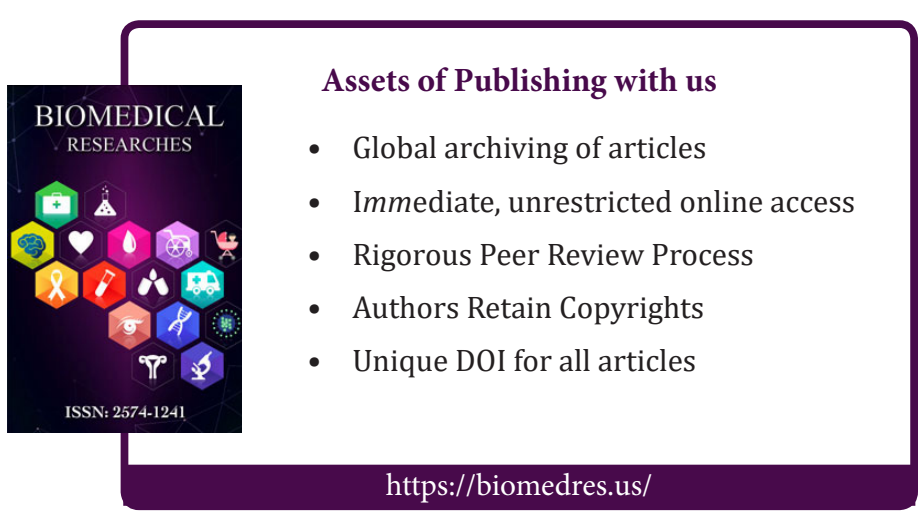

